\title{
El constructivismo cibernético como metateoría educativa: aportaciones al estudio y regulación de los procesos de enseñanza y aprendizaje
}

\author{
Clara Romero Pérez \\ Universidad de Huelva \\ claraduhu.es
}

\section{Constructivismo y Cibernética de segundo orden: postulados teóricos}

El constructivismo implica una ruptura epistemológica respecto al estatuto del conocimiento en la ciencia positivista. De ello se deduce, entre otras premisas, que lo que se va a poner en tela de juicio por parte del constructivismo son las nociones de objetividad, causalidad y realidad y, con ello, las nociones de sujeto y objeto. La emergencia de esta epistemología no puede entenderse sin el desarrollo previo de la teoría sistémica, la primera cibernética y la epistemología genética piagetiana. Al mismo tiempo, los principios que la sustentan no pueden explicarse sin el concurso de la teoría de la complejidad y sus implicaciones en la teoría sistémica, así como también de la cibernética de segundo orden y del espacio interdisciplinario que integran las ciencias cognitivas.

Considerando que ambas teorías pueden converger en un espacio teórico común -que aquí recibe el nombre de constructivismo cibernético- que incluye nociones y conceptos lo suficientemente abstractos y generalizables como para poder ser aplicados al estudio de fenómenos y sistemas complejos, tal es el caso de la educación, esta perspectiva teórica puede concebirse a modo de metateoría o epistemología nuclear desde la que explicar y reformular nuestro objeto de conocimiento en el ámbito de las ciencias de la educación y, aún con mayores posibilidades, en Teoría de la Educación.

El constructivismo incluye la mirada sistémica, esto es, relacional, procesual y molar a la que añade la noción de matriz constructiva. Por emplear un símil o metáfora que sea más ilustrativa, la representación constructivista de la realidad proviene de la relación de continuidad y de circularidad que se da entre el sujeto (y su modelo de cognición) y el dato (o producto del conocer) y otros observadores. De este modo, el principio de matriz constructiva implica que:

(a) la realidad no es externa al sujeto, sino que

(a.1) es una construcción individual que

(a.1.1) se construye a partir de las relaciones interactivas entre sujeto y entorno.

Lo que implica que:

(b) la realidad es co-construida mediante este juego interactivo.

Lo real, desde la perspectiva constructivista, es inmanente al sujeto al participar activamente en la construcción de la experiencia. Pero, al mismo tiempo que no se puede neutralizar al sujeto (observador) en el producto del conocer (realidad, dato, saber), 
tampoco podemos neutralizar a los otros sujetos (co-observadores) que intervienen asimismo en este proceso de co-construcción de la realidad. De ahí que:

(c) la realidad sea co-construida subjetiva e intersubjetivamente mediante procesos de reorganización y reestructuración cognitiva. Dicho proceso afecta tanto a los esquemas conceptuales como de acción -de acuerdo con la epistemología constructivista piagetiana- mediante la puesta en marcha de procesos de reelaboración de sentido y de interpretaciones.

Partiendo de este mismo supuesto, la "no objetividad" de la realidad en el sentido constructivista del término "objetividad", esto es, independiente del sujeto -principio en el que se ha inspirado la ciencia positivista clásica-, lo real no existe o es, sino que emerge. Se deduce de ello que la realidad es, en cierto modo, para los constructivistas el horizonte de la cognición humana y que como todo horizonte no puede ser ultimado o concluido. Lo único real se da en el proceso mismo por el que se hace emerger dicha realidad, o expresado en otros términos, en el propio proceso del conocer. En este contexto explicativo es donde se ubica la aportación de la biología del conocimiento que encuentra en Maturana (1996), Varela (1998) y Bateson (1993) tres de sus representantes más destacados. La propuesta constructivista, en este sentido, indicaría que la realidad sería:

(a) la búsqueda de un argumento convincente (Maturana)

(b) la incorporación a un mundo compartido (Varela; Bruner)

(c) Apariencias, ilusión (Bateson; von Glasersfeld)

De estos presupuestos se puede desprender que:

(d) desde el punto de vista del observador, lo real es producto de un observador. Luego, habrá tantas realidades como observadores haya (subjetivismo), y que:

(e) puede dar lugar a una red ilimitada de realidades que se entrecruzan dando lugar, incluso, a realidades paradójicas u opuestas, pero pueden ser resueltas por la presencia de más observadores co-creando un marco de referencia consistente. La validación del conocimiento se lograría, de este modo, por el grado de consistencia interna alcanzado por las estructuras existentes de conocimiento y el consenso social de los observadores (Feixas y Villega, 2000, 21). De ahí que desde el constructivismo se entienda que racionalizar lo real exige necesariamente la presencia de, al menos, dos observadores para la construcción de marcos de referencia consistentes.

El constructivismo epistemológico resuelve el problema de la objetivación de la realidad incluyendo las representaciones (constructos, interpretaciones, descripciones) de diversos observadores. Aquello que convenimos como realidad aparentemente externa al sujeto y de la que podemos extraer, mediante nuestras observaciones, cierto orden, cierta estabilidad, requiere, como mínimo, la presencia de dos observadores en interacción que van creando y haciendo emerger un marco de referencia consistente (von Foerster, 1996).

El carácter interdisciplinar que ofrece el constructivismo epistemológico ha influido asimismo en la revisión de algunas nociones claves en la Cibernética. Las implicaciones constructivistas de la cibernética se aprecian en los nuevos modelos explicativos que ofrece la Cibernética de segundo orden ("cibernética de la cibernética") respecto de la teoría cibernética clásica. Las nociones de regulación y de información -conceptos centrales en la Cibernética- son revisados desde la cibernética de segundo orden de acuerdo con los postulados constructivistas. Ambas nociones y, junto a ellas, las de cognición y conocimiento, nos permitirán reformular algunos principios y conceptos claves en el ámbito propiamente pedagógico. 
Como paso previo a este análisis pedagógico, se ofrece inicialmente una síntesis conceptual de las nociones anteriormente referidas. En primer lugar, nos referiremos a las ideas de cognición, conocimiento e información. En segundo lugar, nos centraremos en las nociones de autorreferencia y de acoplamiento estructural tomadas del campo de la Biología. La cibernética de segundo orden asumirá estos dos últimos principios procedentes de las propuestas teóricas de la cibernética constructivista del epistemólogo cibernetista vienés Heinz Von Foerster.

\subsection{Acerca de las nociones de cognición, el proceso de conocimiento y el papel de la información}

Los modelos cognitivos que se inspiraron en el conexionismo definían la cognición como un proceso de tratamiento y procesamiento de información.

Desde el punto de vista educativo, el primer corolario que se deduce de las propuestas conexionistas es que la eficacia cognitiva, el comportamiento inteligente, se resuelve mediante la puesta en marcha por parte del sujeto de complejos procesos mentales superiores implicados en la resolución de problemas. La información presupone la consideración de una realidad independiente del sujeto a la que éste puede acceder mediante la puesta en práctica de determinadas competencias y habilidades cognoscitivas complejas. Así pues, dado que existe un saber-objetivo, éste puede ser enseñado a condición de que el sujeto adopte una posición activa en el propio proceso de aprendizaje. El aprendizaje deviene en este contexto una actividad extremadamente compleja que requiere del concurso tanto de las dimensiones cognitivas o mentales como de las afectivo-emocionales propiamente dichas (atención, motivación, representación de un problema, memoria, esquemas cognitivos, etc.). El aprendizaje es un proceso dinámico e implica la selección y organización de la información por parte del sujeto. Desde estos supuestos cognitivistas, el educador debe procurar hacer accesible, inteligible, el objeto de enseñanza. La transmisión de la información no inspira, en ningún caso, a este modelo, sino el hecho de organizar y estructurar dicha información ordenando sus elementos esenciales, a fin de que los alumnos puedan retenerla y aprenderla. El saber-objeto, en cualquier caso, puede ser enseñado por parte del educador.

El constructivismo plantea otros postulados diferentes a los modelos cognitivistas. La cognición es un proceso de atribución y articulación del sentido -que coincidiría en este sentido con los presupuestos de la fenomenología de Gadamer- de una realidad que no está previamente dada, sino que emerge (enactúa) y permite ingresar en un mundo compartido o, por el contrario, permite configurar uno nuevo. El cerebro, la mente vendría a ser un órgano, según las tesis enactivas o constructivistas, que construye mundos, realidades, en lugar de representarlas. Esta forma de entender la cognición humana se refleja en la siguiente afirmación que tomamos de Varela $(1998,109)$ : "La cognición es una acción efectiva: historia del acoplamiento estructural que enactúa (hace emerger) un mundo (...)".

Por lo que respecta a la traducción pedagógica de este enunciado, el aprendiz no es un sujeto aislado, acontextual, sino una entidad que interactúa con su entorno y que es capaz de generar un entramado de relaciones a partir de un contexto que otorga significados mediante una red de intercambios de información que pautan y orientan las conductas de respuesta y emisión de forma continua. La cognición cumple una función básica para la supervivencia del sujeto, al permitirle en este caso no tanto reaccionar a los estímulos del entorno sino organizar su mundo experiencial. La cognición no es independiente de las emociones, de la historia del sujeto, sino que éstas últimas la hacen posible.

Conocido y agente cognitivo se definen mutuamente, no pueden excluirse, son correlativos, están codeterminados. Desde esta perspectiva, la función de la cognición 
supone la capacidad para ingresar en un mundo compartido. Al mismo tiempo, la cognición, al operar con descripciones que superan una simple correspondencia directa con la realidad, implica una reordenación por parte del sujeto de dichas descripciones y no de los objetos en sí mismos (von Foerster, 1996). Algunas de las aplicaciones o incidencias del enfoque constructivista o enactivo se han dado en la teoría de la comunicación. Desde esta perspectiva, la comunicación no consiste en la transferencia de información entre emisor y receptor, sino que por el contrario supone un proceso de modelación mutua de un mundo compartido mediante el lenguaje que permite al hombre conferir existencia a la realidad. La información no supone, en este sentido, la representación de ningún orden dado, externo al sujeto; antes al contrario, la información (el dato), al no referirse a propiedades "objetivas" de la realidad, emerge a partir del propio proceso de conocer, a partir de la actividad cognitiva en sí misma.

\subsection{Acerca de las nociones de autorreferencia y acoplamiento estructural}

La noción de sistema autorreferencial remite a la autonomía cognitiva del sistema/sujeto y ésta, a su vez, a la complejidad del propio sistema/sujeto. La autorreferencia es una operación que se toma a sí misma como objeto (se trata de una operación sobre la operación). Nuestra condición autorreferencial nos permite, por así decirlo, tomar conciencia de nuestra propia conciencia. La autorreferencia por tanto remite a un sujeto (o sistema) que puede remitirse a sí mismo; esto es, que puede así conocer que conoce.

La inclusión de conceptos de segundo orden y de la complejidad por parte de la Cibernética probablemente no nos ayude a resolver los problemas que se plantean hoy a la educación, pero propone a mi juicio una nueva forma de encarar o representar las cuestiones educativas a partir de nociones de segundo orden, esto es, tomando en consideración no sólo lo observado sino también las descripciones del observador.

Aplicando conceptos de primer orden, el aprendizaje sería el resultado de una acción reguladora desde fuera por parte del agente educativo. Es al docente a quien compete seleccionar los estímulos del medio inductores del cambio en el operar del sujeto. La cibernética de segundo orden no niega que esto sea posible para aprendizajes que no sean excesivamente complejos, pero afirma que los procesos cognitivos superiores sólo pueden ser especificados por el propio sujeto a partir de su propia dinámica estructural que determina, como subraya Asensio Aguilera $(1998,186)$, los cambios que tienen en él lugar y los estímulos del medio que pueden inducir dichos cambios. Resulta así que el aprendizaje es el resultado del acoplamiento estructural (Maturana, 1996; Varela, 1998) del sujeto con su entorno (organismo y medio), o sea, del encaje o compatibilidad alcanzada entre el sujeto y su entorno y supone, al mismo tiempo, la transformación de la propia organización cognitiva del sujeto. El desarrollo no se genera o es inducido desde fuera del organismo (sujeto), actuando el medio como seleccionador (natural) de los organismos, sino de la interacción sujeto y medio para que sea posible una reorganización del sistema.

\section{Redefinir la actividad pedagógica: hacia una pedagogía orientada a promover la autonomía del estudiante.}

Las incidencias que el constructivismo plantea a la tecnología de la enseñanza son, como veremos, claras y notorias. En primer lugar, disloca uno de los ejes sobre los que se ha hecho pivotar a los procesos de enseñaza-aprendizaje: el saber y la regulación o conducción de los aprendizajes mediante el empleo de tecnologías de control heterónomo (reductoras de complejidad). Como tendremos oportunidad de analizar a continuación, la simbiosis constructivista-cibernética se apoyará, por el contrario, en otros ejes:

- la autonomía del sujeto y la relativización del saber, enfatizando la génesis y desarrollo 
del proceso del conocer;

- una nueva orientación de la regulación pedagógica que, en este caso, no es inducida desde el medio sino autorregulada y generada por el propio sujeto.

Una propuesta en este sentido es la que formula Georges Lerbet (1995) y que expone como nuevo modelo de la ingeniería en el ámbito pedagógico: facilitar las condiciones que posibiliten en cada alumno la creación de un sistema personal de autorregulación (pilotaje) del aprendizaje. Desde este punto de vista se trataría de ofrecer un contexto educativo diversificado, no restrictivo ni lineal, de tal modo que los aprendizajes más complejos pudieran ser viables a partir del propio entorno de aprendizaje.

La enseñanza no debe obviar la condición autorreferencial del aprendiz y, por tanto, su capacidad para crear consistencias. La condición humana se basa precisamente en la doble dimensión de la conciencia: constituida por datos, informaciones, señales, que informan de una realidad constituida intersubjetivamente, pero también es autoconciencia; esto es, constituyente de realidad y con capacidad para generar significados; esto es, que es capaz de atribuir sentido a sí mismo y a su entorno.

La cibernética de primer orden sólo operaba con conceptos y elementos de primer orden, esto es, los que se refieren a los objetos excluyendo la condición autorreferencial del sujeto. En consecuencia, los aprendizajes pueden ser definidos a priori, externamente al aprendiz por el educador. Los aprendizajes operan con saberes que sintetizan los aspectos relevantes de una realidad aparentemente externa al sujeto.

El saber se refiere a un saber sobre algo. El educador operaría con un producto previamente organizado. Se trata de un saber-objeto (sin sujeto) independiente del sujeto. El saber se confunde con conocimiento y, una vez organizado y estructurado de acuerdo con las características psico-evolutivas del sujeto y los modos de procesar la información, está listo para ser puesto en circulación. El problema radica a mi juicio en que no se establece una diferenciación entre saber y conocimiento y se olvidan las relaciones entre ambos. Von Foerster $(1996,189)$ señala que información y conocimiento son dos caras de la misma moneda que no pueden excluirse y que en ningún caso pueden ser transmitidos, pues remiten a procesos.

Desde su posición niega que las instituciones educativas sean las depositarias del conocimiento ni del proceso por el que éste se genera: la información, y así lo señala cuando indica que "las universidades no son depositarias de conocimiento que se transmite de generación en generación, ya que tu actividad nerviosa es tu actividad nerviosa y, por tanto, no es la mía".

En todo caso, de acuerdo con este autor, lo que resultaría pertinente es reservar para la institución educativa o el agente educativo el término de mediadores o portadores de información potencial, esto es, generadores de procesos que conducen, a su vez, al estudiante a crear y apropiarse, en definitiva, él mismo, de su propio conocimiento que, en definitiva, es un proceso intransferible. En este sentido estas tesis coinciden, en cierto modo, con las formulaciones que en las décadas de los 70 y 80 ofreció Carl Rogers en su propuesta de una educación centrada en el estudiante y que incorporaba la idea de conocimiento como un proceso de descubrimiento intelectual y emocional que no se agota en sí mismo sino que se extiende a la apertura de nuevos aprendizajes. El conocer implica saber, pero es un proceso más abarcativo que incluye a un sujeto que ha aprendido "cómo aprender lo que desea saber" (Rogers, 1995, 141).

El constructivismo cibernético nos aproxima a dimensiones que hasta ahora tendían a ser neutralizadas o trivializadas tanto desde las propuestas teórico educativas como desde el ejercicio de la acción pedagógica. Una de las dimensiones que, hasta el momento, se 
habían neutralizado es la referida a la complejidad; otra de las dimensiones neutralizadas en la enseñanza son las realidades de segundo orden que no son accesibles a los observadores (profesores, administradores, estudiantes, etc.) si no es mediante un esfuerzo inquisitivo, autorreflexivo y dialógico.

\section{1. La neutralización de la complejidad}

Complejidad, de antemano, no significa "complicado" o "difícil". Este término denota por así decirlo la multidimensionalidad de aspectos, elementos y relaciones entre elementos entre los cuales se encuentra el propio observador- desde el que podemos distinguir y apreciar un fenómeno dado. Complejidad, desde mi punto de vista, no supone negar la posibilidad de estudio de dicho fenómeno, como puede ser, en nuestro caso, la educación, sino de clarificarlo, explicarlo, por medio de métodos adecuados a dicho objeto. Por tanto, el que un fenómeno como el educativo -en tanto actividad, institución o acciónpresente como características esta dimensión o propiedad no nos exime de su estudio ni tampoco quiere decir y, con ello convengo con el planteamiento desarrollado por Gros Salvat (1996) que la pedagogía sea una ciencia imposible.

Probablemente, esta neutralización de la complejidad sea debido al modo como hemos racionalizado nuestro objeto. Se extendió la creencia que sólo la racionalidad de la ciencia objetivista era la única vía certera, fiable, para acceder a la realidad, excluyendo otras, sin caer en la cuenta que ni certeza significaba exactitud ni tampoco precisión. Las ciencias cognitivas han señalado que la racionalidad es el producto de una operación cognitiva y meta-cognitiva que una comunidad enraizada, a su vez, en una tradición, realiza y a la que aplica un método formalizado y universal. Sin embargo, constata el constructivismo que mediante esta operación de racionalización de lo real, propio del método científico positivista-experimental, sólo se podía acceder a realidades de primer orden, esto es, realidades en las que se presupone existe una separación entre observador y fenómeno observado cuyo puente de unión sería el método científico. Esta crítica se hace extensiva también a la Teoría de Sistemas que, aunque en efecto, incluía una perspectiva relacional para analizar los sistemas, no obstante, no incluía al observador en el sistema estudiado.

De esta argumentación se desprenden tres corolarios:

- La imposibilidad de aprehender mediante una racionalidad disyuntiva los fenómenos complejos. La educación comparte, precisamente, este carácter y por ende, requiere ser analizada desde esta racionalidad.

- La posibilidad del conocimiento científico de fenómenos complejos, como el fenómeno educativo, incluyendo la dualidad que subyace en toda unidad de conocimiento (la del observador frente al objeto y la del observador frente a sí mismo).

- La imposibilidad de disociar al observador del objeto observado.

Interpreto que más allá de un discurso fácil o, por el contrario, alarmista respecto de las posibilidades del quehacer científico o la imposibilidad de lograr una realidad fundamentante que nos oriente, la ruptura constructivista exige la libertad del sujeto, su autonomía y que dicha capacidad de autonomía sólo puede lograrse si él (en tanto observador) se hace objeto de su propio observar y es capaz de trascender, como propondría el constructivismo, las restricciones de su propio mirar a través de la mirada de otros observadores. Creo, sinceramente, que las consecuencias éticas que se podrían desprender de los enunciados constructivistas cibernéticos son, a mi juicio, interesantes desde el campo de la investigación y de la práctica pedagógica.

\subsection{La neutralización de las realidades de segundo orden}


Señalábamos que una realidad de segundo orden es el producto de una operación de segundo orden, sin la cual no podríamos acceder a conocerla. Una operación de segundo orden es una operación que toma como objeto la operación en sí misma. Algunas implicaciones que se desprenden para la práctica educativa al incluir -y, por tanto, no neutralizar- estas realidades de segundo orden sería la de admitir diversos puntos de vista en el planteamiento de un problema o en la formulación de pautas de acción. Aunque podría parecer que con ello nos introducimos en un relativismo estéril, aplicando los principios del constructivismo cibernético, esta premisa se resuelve, de acuerdo con von Foerster (1996), inscribiéndola en este marco metateórico:

(2.1.1) Sin comunicación no hay regulación;

(2.1.1.1) Sin regulación no hay meta;

(2.1.1.3) Sin una meta, el concepto de sociedad o de sistema se vuelve vacío;

(2.1.1.4) La competencia implica responsabilidades;

Los cibernetistas se apoyan, como sabemos, en los bucles de retroalimentación como operaciones que sirven para regular la dinámica de los sistemas. La pérdida de este bucle puede conducir a la pérdida del control del sistema $\mathrm{y}$, por consiguiente, la desestructuración de éste. El mensaje constructivista cibernético subraya la necesidad de articular un lenguaje plural (interdisciplinar), combinar posturas, planteamientos, enfoques; abandonar una postura egocéntrica o unilateral en la formulación del problema exhortando a todos los observadores a problematizar previamente el problema antes de resolverlo.

Sin duda, esto implica un sólido ejercicio de racionalidad que no elimina la irracionalidad, el error o, si se me permite también, el engaño -también estos forman parte de la condición humana-, sino que las integra, para después neutralizarlas o eliminarlas, emprendiendo el curso al que obliga la racionalidad humana que no es sólo, como advierte Morin (1999), teórica ni crítica, sino también autocrítica.

El valor del constructivismo cibernético como metateoría reside en su grado de abstracción y, por tanto, su posibilidad de ser aplicados a diferentes contextos. La reformulación de algunos conceptos básicos, directamente vinculados con la realidad educativa pueden ayudarnos a explicar, a partir de este nuevo enfoque, de qué modo podemos optimizar nuestros sistemas educativos, los procesos de enseñanza y aprendizaje y reflexionar, ubicándonos en estas realidades de segundo orden, acerca de nuestro papel activo y no sólo reactivo o condicionado, en la organización y estructuración de nuestra relación en el medio. Incluso podría desprenderse de esta metateoría, sin caer por ello en idealizaciones falsas y acríticas, un modelo ético que apela a la responsabilidad del hombre en el desempeño de su acción y el principio ético de la colaboración, la cooperación y la comunicación como bases de un nuevo modelo para la regulación de un espacio común y plural. ¿Tal vez no sea éste un modelo que se adapta a las exigencias democráticas de nuestro tiempo?.

\section{Bibliografia}

ASENSIO AGUILERA, J. Ma . (1998) Aprendizaje de la complejidad y nuevas tecnologías, Addenda presentada al XVII SEMINARIO INTERUNIVERSITARIO DE TEORÍA DE LA EDUCACIÓN La educación universitaria: nuevos retos educativos y tecnológicos, Málaga, 16-18 de Noviembre, (documento policopiado), 185-189.

AZNAR MINGUET, P. (Coord.) (1999) Teoría de la educación. Un enfoque constructivista. Valencia, Tirant lo Blanch.

CEBERIO, M. y WATZLAWICK, P. (1998) La construcción del universo. Conceptos introductorios y reflexiones sobre epistemología, constructivismo y pensamiento sistémico. Barcelona, Herder. 
COLOM CAÑELLAS, A. J. (1997) La regionalización de la educación como tecnología cognitiva virtual, Teoría de la Educación. Revista Interuniversitaria, 9, 7-19.

FEIXAS, G. y VILLEGAS, M. (2000) La epistemología constructivista, en FEIXAS, G. y VILlEGAS, M. Constructivismo y psicoterapia ( $3^{\text {a }}$ edición). Bilbao, Desclée de Brouwer, 29-102.

GARCÍA CARRASCO, J. y GARCÍA DEL DUJO, Á. (1995) Epistemología Pedagógica (I), Teoría de la Educación. Revista Interuniversitaria, 7, 5-38.

GARCÍA CARRASCO, J. y GARCÍA DEL DUJO, Á. (1996) Epistemología Pedagógica (II), Teoría de la Educación. Revista Interuniversitaria, 8, 5-42.

GARCÍA DEL DUJO, Á. (1992) Constructivismo e intervención pedagógica: a propósito de quien construye, Teoría de la Educación. Revista Interuniversitaria, 4, 127-138.

GROS SALVAT, B. (1996) Pensar sobre la educación desde una concepción sistémicocibernética, Teoría de la Educación. Revista Interuniversitaria, 8, 81-94.

GROS SALVAT, B. (coord ${ }^{\mathrm{a}}$.) (1997) Diseños y programas educativos. Pautas pedagógicas para la elaboración de software. Barcelona, Ariel.

LERBET, G. (1995) Les nouvelles sciences de l'éducation. Paris, Éditions Nathan.

MATURANA, H. (1996) La realidad: ¿Objetiva o construida?. Fundamentos biológicos del conocimiento, Vol. II. México, Anthropos/Universidad Iberoamericana/ITESO.

MORIN, E. (1999) Los siete saberes necesarios para la educación del futuro. Paris, UNESCO.

MORIN, E. (2001) La mente bien ordenada. Repensar la reforma. Reformar el pensamiento. ( $2^{\mathrm{a}}$ edición) Barcelona, Seix Barral.

NARDONE, G. y WATZLAWICK, P. (1999) Introducción, en NARDONE, G. y WATZLAWICK, P. Terapia breve: filosofía y arte. Barcelona, Herder.

PERRENAUD, PH. (1996) Enseigner: agir dans l'urgence, décider dans l'incertitude. Paris, ESF Éditeur.

SCHÖN, D.A. (1998) A la recherche d'une nouvelle épistémologie de la pratique et de ce qu'elle implique pour l'éducation des adultes, en BARBIER, J. M. (dir.) Savoirs théoriques et savoirs d'action. Paris, Presses Universitaires de France, 201-222.

VARELA, F. J. (1998) Las ciencias cognitivas: tendencias y perspectivas. Cartografía de las ideas actualesa. Barcelona, Gedisa.

VÁZQUEZ GÓMEZ, G. (1991) La Pedagogía como ciencia cognitiva, Revista Española de Pedagogía, 188, 123-146.

VÁZQUEZ GÓMEZ, G. y BÁRCENA ORBE, F. (1999) Pedagogía Cognitiva: la educación y el estudio de la mente en la Sociedad de la Información, Teoría de la Educación. La Educación y la cultura en la Sociedad de la Información, 1, http://www3.usal.es/teoriaeducacion/

VON FOERSTER, H. (1996) Las semillas de la cibernética. Obras escogidas. Barcelona, Gedisa.

(C) Ediciones Universidad de Salamanca 